\title{
A 12-Week Maintenance Therapy with a New Prepared Viscous Budesonide in Pediatric Eosinophilic Esophagitis
}

\author{
Salvatore Oliva ${ }^{1}$ (D) Danilo Rossetti ${ }^{1} \cdot$ Paola Papoff $^{2} \cdot$ Antonio Tiberti $^{3} \cdot$ Saverio Mallardo $^{1} \cdot$ Danila Volpe $^{1}$. \\ Cosimo Ruggiero ${ }^{1}$ - Giusy Russo ${ }^{1}$ - Debora Vezzoli ${ }^{1}$ - Sara Isoldi ${ }^{1} \cdot$ Salvatore Cucchiara ${ }^{1}$
}

Received: 10 July 2018 / Accepted: 29 December 2018 / Published online: 19 January 2019

(c) The Author(s) 2019

\begin{abstract}
Background A new prepared oral viscous budesonide (PVB) has been effective in inducing clinical and histological remission in pediatric eosinophilic esophagitis (EoE).

Aims To evaluate the efficacy of a 12-week maintenance therapy on clinical, endoscopic, and histological remission using half of the dose used in the induction therapy.

Methods We prospectively enrolled pediatric patients with active EoE. After 12 weeks of induction therapy with PVB $(<150 \mathrm{~cm}: 2 \mathrm{mg} /$ day; $\geq 150 \mathrm{~cm}: 4 \mathrm{mg} /$ day) patients received a maintenance dose of half of the dose used in the induction therapy ( $1 \mathrm{mg}$ or $2 \mathrm{mg}$ ) for another 12 weeks. A 12-week follow-up was then performed in all patients after the end of therapy. Endoscopy was performed at weeks 0,12, 24, and 36. Symptoms, endoscopy, and histology scores were also calculated. Serum cortisol was evaluated during the treatment period.

Results We enrolled 20 children (15 males; median age 10 years; range 4-17). After the 12-week induction therapy 18 patients $(90 \%)$ were in remission, with a significant decrease in the median peak of eosinophil count/HPF as well as a marked reduction in clinical, endoscopic, and histological scores $(p<0.01)$. At the end of the maintenance therapy (week 24), 17 patients (85\%) were still in remission, while there were only 9 at week 36 (45\%). No significant changes in cortisol levels were observed during the study period.

Conclusions The 12-week maintenance treatment with the half the dose of PVB was effective in sustaining remission at week 24; however, no reduction in the rate of relapse after suspension of treatment occurred.
\end{abstract}

Keywords Eosinophilic esophagitis $\cdot$ Oral viscous budesonide $\cdot$ Elimination diet $\cdot$ Pediatric endoscopy $\cdot$ PPIs

\section{Abbreviations}

EoE Eosinophilic esophagitis

OVB Oral viscous budesonide

PVB Prepared viscous budesonide

MII-pH $\quad \mathrm{pH}$ multichannel intraluminal impedance

Salvatore Oliva

salvatore.oliva@uniroma1.it

1 Department of Pediatrics, Pediatric Gastroenterology and Liver Unit, Sapienza University of Rome, Viale Regina Elena 324, 00161 Rome, Italy

2 Department of Paediatrics, PICU, Sapienza University of Rome, 00161 Rome, Italy

3 Department of Radiological Sciences, Oncology, and Anatomical Pathology, Sapienza - University of Rome, Rome, Italy

\section{Introduction}

Eosinophilic esophagitis (EoE) is a chronic immune-/ antigen-mediated disorder of the esophagus characterized histologically by eosinophil-predominant mucosal inflammation and clinically by symptoms of esophageal dysfunction [1]. Its incidence in children has been increasing and is currently similar to that of pediatric inflammatory bowel disease (IBD) [2]. EoE involves progressive esophageal remodeling that can lead to esophageal dysmotility and strictures, if untreated [3,4]. Therapeutic targets currently include improvement of symptoms, remission of histopathological features, and prevention of long-term complications. However, no approved therapies for pediatric EoE exist to date [5].

Because of the chronic course of the disease, with a tendency to relapse within a few months after therapy withdrawal and to develop progressive fibrosis over time 
$[6,7]$, long-term therapy for EoE is needed. Although diet is widely seen as a valuable maintenance strategy in pediatric EoE [8], its long-term tolerance by children has been challenged [9]. Topical corticosteroids have been shown to be effective in children, as demonstrated by several studies, but their efficacy or safety profile for chronic use is still in infancy [10]. A few studies have demonstrated that a maintenance therapy with swallowed fluticasone in children leads to a sustained improvement of eosinophilic infiltrations of the esophagus [11, 12]. Long-term efficacy of oral viscous budesonide (OVB) has been assessed only in adults, and no data are available in pediatric patients $[13,14]$. OVB is actually considered more effective than the nebulized suspension, due to the more prolonged esophageal mucosal contact time [15]. Unfortunately, no OVB specifically designed and approved for esophageal drug therapy is currently available, and options are limited to off-label administering of nebulized budesonide mixed with slurry sweeteners [16]. This leads to a histological non-response rate ranging from 25 to $50 \%$ in clinical trials and presumably even higher in the real world [17]. Recently, we have demonstrated the efficacy of a new prepared formulation of OVB (PVB) in inducing remission of pediatric EoE [18]. In this study, we aimed to determine the efficacy of PVB in a 12-week maintenance phase (using a 50\% dose reduction from the induction dose) to sustain remission of pediatric EoE. The improvement in symptom, endoscopy, and histology scores, as well as the safety profile of this formulation was evaluated.

Fig. 1 Flowchart of the study

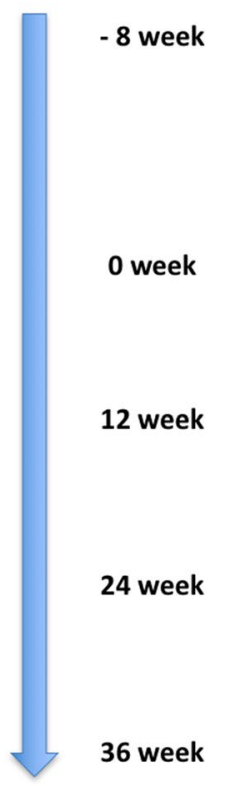

\section{Materials and Methods}

\section{Study Design}

This is a prospective, single-site, pilot study of 12 -week maintenance therapy with PVB in children with EoE. After 12 weeks of induction therapy, patients with histological and clinical remission received half the dosage for an additional 12 weeks, for a total period of 24 weeks. A final evaluation at 36 weeks was performed, 12 weeks after the end of treatment (Fig. 1).

\section{Patients}

Eligible patients were recruited at the Pediatric Gastroenterology and Liver Unit of the Sapienza University of Rome during a 12-month period (between December 2014 and November 2015). This Unit is a tertiary referral pediatric center for gastrointestinal (GI) eosinophilic disorders. We prospectively enrolled consecutive pediatric subjects with active EoE (either new or established). Inclusion criteria were as follows: age $<18$ years; diagnosis of EoE with $>15$ eosinophils/high-power field (HPF) in esophageal biopsies after a PPI trial of at least 8 weeks, according to the 2014 ESPGHAN guidelines [7]; requesting alternatives to diet for new diagnosis, or a different pharmacologic treatment during a histological flare (in case of previous diet treatment and/or nebulized or systemic steroids). Exclusion criteria were as follows: the presence of non-EoE GI diseases (eosinophilic gastroenteritis/colitis, IBD, or celiac disease); esophageal stricture on baseline endoscopy that precluded passage of an

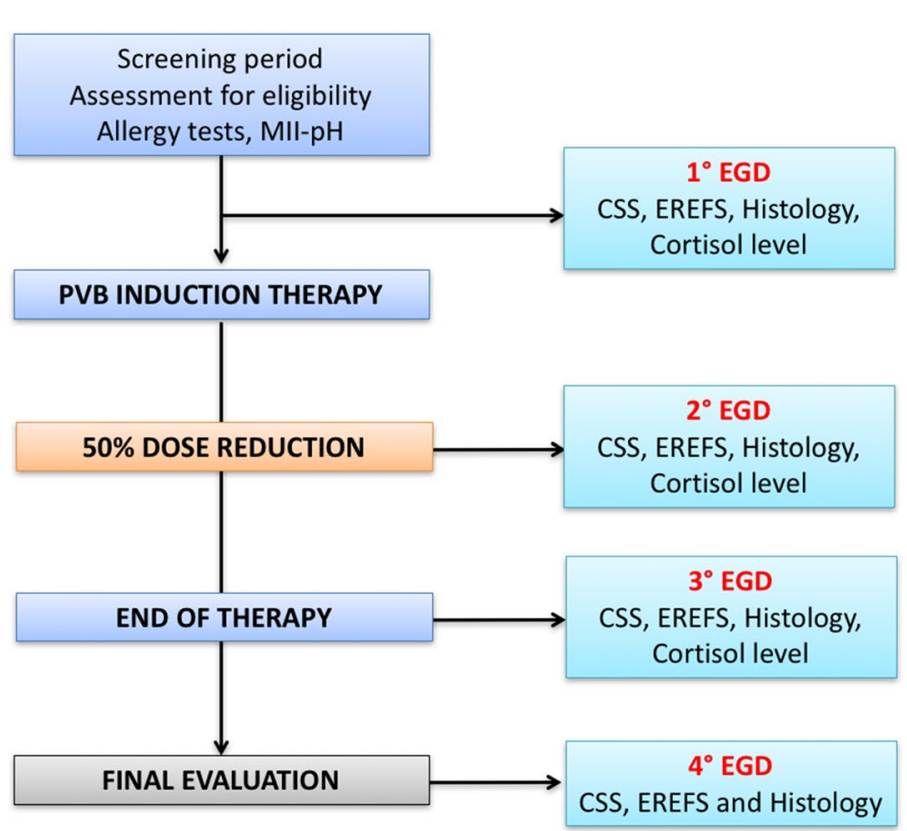


upper scope; use of steroids (topical or systemic) or elimination diet for EoE within 4 weeks of the baseline endoscopy; change in PPIs dosing regimen, diet, allergy medications, or inhaled steroids during the study period. Written informed consent was obtained from parents of all children; children over 12 years of age signed a statement of assent.

An allergy evaluation (through skin prick test, atopy patch test, and specific IgE tests) was performed in all patients before the enrollment. A 24-h pH multichannel intraluminal impedance (MII-pH) was also performed in the absence of any therapy and during the screening period (or before an 8-week PPI therapy for new diagnosis) in order to reduce the potential effect of concurrent GERD on esophageal eosinophilia in case of positive results. According to the ESPGHAN guidelines, the following MII-pH variables were analyzed: occurrence of both acid (defined as a drop in $\mathrm{pH}<4.0$ ) and non-acid (defined as a drop in $\mathrm{pH}>7.0$ ) GER events; total exposure acid time (\% of GER); the total number of acid and non-acid GER events; the long-lasting number of acid GER events; the symptomatic index (SI), that is the percentage of symptoms attributable to both acid (SI-A) and non-acid (SI-NA) GER events. An examination was considered positive only if at least $\geq 3$ of variables reported above were positive. Patients with a positive MII$\mathrm{pH}$ or with evidence of erosive esophagitis at index endoscopy continued a concurrent PPI therapy (after the PPI test of 8 weeks in new diagnoses) at dose of $1.0 \mathrm{mg} / \mathrm{kg} /$ day along the study. All authors have had access to the study data and have reviewed and approved the final manuscript. The study protocol was defined in accordance with the Declaration of Helsinki and approved by the Ethical Committee of the University Hospital Umberto I in Rome (ref. 957/14).

\section{Treatment}

PVB is a prepared formulation of budesonide, mainly mixed with xylitol, at a concentration of $0.2 \mathrm{mg} / \mathrm{mL}$, made by ITC Farma Srl (Pomezia, Italy). This company provided the entire drug supply used in this study. Participants were allocated according to their height. Patients $<150 \mathrm{~cm}$ and $\geq 150 \mathrm{~cm}$ height received an induction therapy of $1 \mathrm{mg} / 5 \mathrm{ml}$ bid ( $2 \mathrm{mg} /$ daily) and $2 \mathrm{mg} / 10 \mathrm{ml}$ (4 mg/daily), respectively, for 12 weeks. After the induction therapy with PVB, patients were followed up at our site for repeat clinical, endoscopic, and histological evaluations. If patients achieved a resolution of their main symptoms and a histological reduction in peak esophageal count of $>50 \%$ from the baseline, PVB was reduced to half the dose for another 12 weeks, while in case of lack of response ( $\geq 20$ eos/HPF) the same dosage was continued. A complete evaluation was repeated at the end of the 12-week maintenance therapy (week 24) and at the end of the study (week 36).
Doses were administered after breakfast and at bedtime, respectively, by using a capacity syringe. The bottle was conserved at room temperature (no $>35^{\circ} \mathrm{C}$ or $\left.<4{ }^{\circ} \mathrm{C}\right)$. Subjects were educated not to eat, drink, rinse their mouth, or brush their teeth for at least 30 min after drug assumption. Patients were monitored at 6-week intervals and had access to a physician over the study period to assist with any issues regarding the trial. Study medication adherence was also evaluated at each study visit by assessing the volume of medication remaining in each bottle. Adherence was calculated by dividing the amount of medication taken by the amount that should have been taken, multiplied by 100 . Subjects were considered adherent if they had received between 80 and $120 \%$ of the expected amount of medication.

\section{Clinical Symptom Score}

Patients were clinically evaluated at 6-week intervals. A clinical score was calculated at baseline, 12, 24, and 36 weeks, considering seven variables: regurgitation/heartburn, abdominal pain, nausea/vomiting, anorexia/early satiety, dysphagia/food impaction, symptom-induced nocturnal awakening, and GI bleeding; each symptom category was scored from 0 to 2 for intensity and frequency, for a total score from 0 to 14 [19].

\section{Endoscopy and Histology}

Endoscopy was performed at baseline, 12, 24, and 36 weeks (3 months after the end of treatment) by the same boardcertified gastroenterologist ( $\mathrm{SO}$ ), using conscious sedation, deep sedation, or general anesthesia (according to different ages) (Fig. 1). Endoscopic alterations were evaluated with the EREFS score that considered six characteristics: rings, white plaques, furrows, decreased vascular pattern (edema), stricture, and esophageal shearing (maximum total score 9 pts) [20]. At the inclusion/T0 endoscopy, all subjects received esophageal, gastric, and duodenal biopsies. At least 2 mucosal pinch biopsies were obtained from the proximal, mid-, and distal esophagus. Subjects with peak intraepithelial eosinophil counts of $\geq 15 / \mathrm{HPF}$ and no significant gastric or duodenal pathology were histologically eligible for the study. During the follow-up endoscopies, at least 2 biopsies from all 3 esophageal levels were performed in all subjects. Gastric and duodenal biopsies were performed at the physician's discretion. Proximal, mid-, and distal esophageal biopsies, taken before and after treatment, were processed routinely and evaluated using light microscopy by an expert pathologist (AT). Two biopsies per level (proximal, middle, and distal) yielded between 15 and 20 evaluable HPFs. A peak eosinophil count/HPF was obtained for each esophageal level by counting the number of eosinophils in the most inflamed area by using a Nikon Eclipse E400 (Nikon, Tokyo, 
Japan) light microscope at $\mathrm{x} 400$ magnification $\left(0.3 \mathrm{~mm}^{2}\right.$ HPF). Histological findings were scored with values of peak intraepithelial eosinophils count and other mucosal alterations characteristics of EoE (including eosinophilic degranulation, eosinophil microabscesses, basal layer hyperplasia dilated intracellular spaces or spongiosis, and lamina propria fibrosis) [21], with a total score of 18 .

\section{Outcome Measures}

The primary outcome was the persistence of histological remission (peak eosinophil count $<6$ eos/HPF) after 12 weeks of dose reduction (week 24) and after 12 weeks of follow-up and no drug (week 36). After the induction and maintenance phases, the response to therapy was determined by comparing baseline and final treatment peak counts/HPF under light microscopy $(\times 400)$. Based on peak eosinophil count, patients were categorized into responders (0-6 eos/ HPF), partial responders (7-19 eos/HPF), and non-responders ( $\geq 20$ eos/HPF). Secondary outcomes included persistence of response in symptom, endoscopy, and histology scores at the end of treatment.

\section{Safety Assessment}

This included adverse event monitoring during the treatment; physical examination; height; weight; vital signs (heart rate, blood pressure); and laboratory tests (complete blood count with differential, serum glucose, morning serum cortisol). Clinical assessments were considered at baseline, $6,12,18,24,30$, and 36 weeks, while laboratory tests at baseline, 12, and 24 weeks only (Fig. 1). Baseline and posttreatment morning cortisol levels were measured to estimate adrenal suppression of the PVB suspension. The cortisol was measured during other laboratory tests.

\section{Statistical Analysis}

All statistical analyses were performed using the SPSS (SPSS 17.0, Chicago, USA). Two-tailed $P$ values were calculated using paired $t$-tests to compare the means of patient values for eos/HPF, endoscopy, histology, and symptom scores before and after PVB therapy. Two-tailed unpaired $t$-tests were utilized in order to compare variables grouped by responders versus non-responders and by relapse versus remission.

Considering an effect size of at least 0.7 between pairs, a sample size of 20 has been considered to achieve a power of $80 \%$ and a level of significance of $5 \%$ (two-sided).

Results with $p$ values $<0.05$ were considered statistically significant. Both mean and median statistics were generated, both were equivalent, and mean statistics are presented. Data were given as mean $\pm \mathrm{SD}$ and/or as median (and ranges).
Table 1 Patients' demographics and clinical information

\begin{tabular}{ll}
\hline Patients & $N=20$ \\
\hline Median age, y (range) & $10(4-17)$ \\
Gender (M, F) & 15,5 \\
Height & \\
$<150 \mathrm{~cm}$ & $8(40 \%)$ \\
$\geq 150 \mathrm{~cm}$ & $12(60 \%)$ \\
New diagnosis & $5(25 \%)$ \\
Disease duration, $m$, median (range) & $17.5(1-42)$ \\
Age at diagnosis, $y$, median (range) & $10.8(4-17)$ \\
Previous medications $[n(\%)]$ & \\
Corticosteroids (any) & $15(75 \%)$ \\
Diet & $5(25 \%)$ \\
Concurrent PPI & $11(55 \%)$ \\
Allergy $[n(\%)]$ & \\
Family history & $8(40 \%)$ \\
RAST/skin prick test & $14(70 \%)$ \\
Atopy patch test & $3(15 \%)$ \\
\hline
\end{tabular}

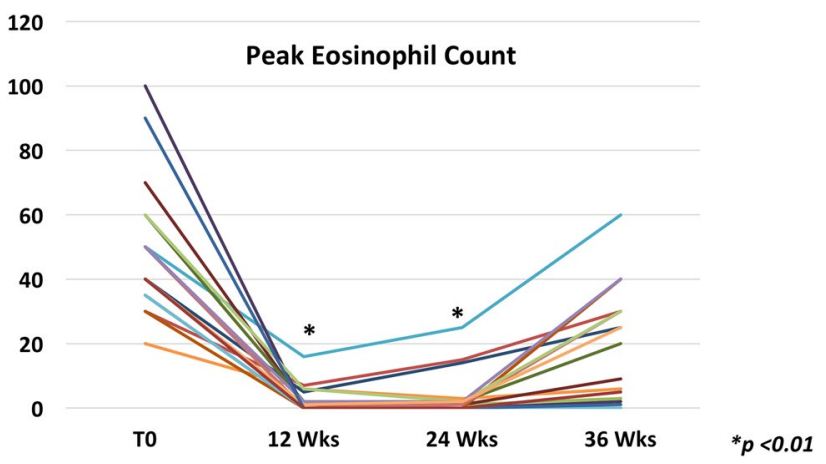

Fig. 2 Values of peak eosinophil count before and after induction and maintenance phases of the treatment with the new suspension of oral viscous budesonide. $* p<0.01$, weeks 12 and 24 compared with the baseline. Week 12 compared with week $24, p=0.19$

\section{Results}

During the study period, 29 patients were screened. Six were excluded because of one or more exclusion criteria, while 3 had a positive response to the 8-week PPI therapy and were considered as PPI-REE (according to the old EoE classification). Thus, 20 patients were enrolled and considered for the statistical analysis. All clinical information is summarized in Table 1.

During the induction phase, the PVB suspension significantly reduced the mean peak eosinophil count/HPF from baseline (50; range $15-100)$ to 12 weeks $(0.5$; range $0-16)(p<0.01)$ (Fig. 2). Eighteen patients $(90 \%)$ were considered as "responders," but two were considered as 


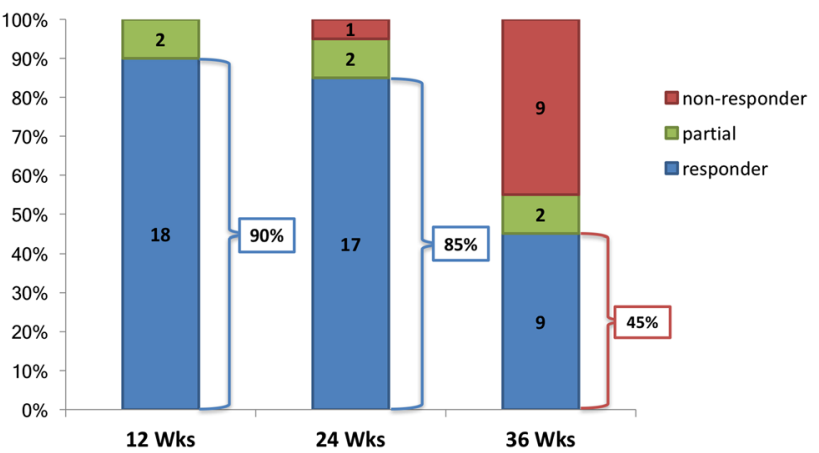

Fig. 3 Percentage of responders after 12- and 24-week treatment and at 36-week follow-up

"partial responders," despite a significant improvement in symptoms. No patients were non-responders (Fig. 3). Eleven $(55 \%)$ were also treated with proton pump inhibitors, including the two partial responders.

All patients were treated with a half-dose PVB therapy for additional 12 weeks. At week 24, the mean value of peak eosinophil count/HPF was 1 (range 0-25) (no significant difference from the 12 -week value). Only 1 patient of 18 responders presented a peak intraepithelial eosinophil count $>6(5 \%)$ after the dose reduction; thus, the overall remission rate was $85 \%$ (Fig. 3). The two partial responders exhibited an increase in the peak eosinophil count ( $>15$ eos/ HPF) despite the absence of symptoms (Fig. 2).

At the end of the follow-up period (week 36), 9 (45\%) patients were still in remission, while 7 (35\%) showed a significant increase in the eosinophil count, and the remaining $1(5 \%)$ showed a partial increase (Figs. 2, 3). The 3 active patients at 24 weeks had worse symptoms and eosinophil infiltration after discontinuation of therapy. In the majority of the patients [5] the relapse started from the distal part of the esophagus, but there was no statistically significant difference from those with a relapse in the mid- or proximal part. No statistically significant difference in any baseline parameter as well as in pre- and post-treatment characteristics was observed between children with a relapse and those without (Tables 2, 3).

Only 14 patients were positive at RAST/SPT tests. Ten were either positive at RAST and SPT, while 4 had only RAST positivity. The most common antigen was cow's milk 8/14 (57\%), followed by egg 6/14 (38\%), fish 4/14 (29\%), soy $3 / 14(21 \%)$, and wheat $3 / 14(21 \%)$. Eight (57\%) had also a sensitization to environmental allergens. No difference was found between response to PVB, tendency to relapse, and positivity at RAST/SPT (Table 2).

The new PVB suspension was markedly effective in improving the mean clinical symptom score from 4.5 (range 0-9) to 0.45 (range 0-7) and to 0.65 (range 0-6) after 12 and 24 weeks of therapy, respectively $(p<0.01)$
Table 2 Baseline characteristics of patients with a relapse compared with those in remission

\begin{tabular}{llll}
\hline & Relapse $(n=11)$ & Remission $(n=9)$ & $p$ value \\
\hline Age, y, mean \pm SD & $11.3 \pm 4.5$ & $10.9 \pm 3.6$ & NS \\
Male $[n(\%)]$ & $9(82)$ & $6(67)$ & NS \\
Months since & $16.5 \pm 13.8$ & $13.7 \pm 12.2$ & NS \\
$\quad$ EoE diagnosis, & & & \\
mean \pm SD & & & \\
Height, cm, mean \pm SD & $146.5 \pm 29.4$ & $150.2 \pm 21.2$ & NS \\
Previous medication & & & \\
$\quad n(\%)]$ & & & \\
Corticosteroids (any) & $8(73)$ & $7(78)$ & NS \\
Diet & $2(18)$ & $3(33)$ & NS \\
Concurrent PPI & $6(55)$ & $5(55)$ & NS \\
Allergy [n (\%)] & & & \\
Family history & $5(46)$ & $3(33)$ & NS \\
RAST/skin prick test & $8(73)$ & $6(67)$ & NS \\
Atopy patch test & $1(9)$ & $2(22)$ & NS \\
\hline
\end{tabular}

(Fig. 4a). A statistically significant increase from the end of therapy was observed at 36 weeks ( 1.85 ; range $0-7$, $p<0.05)$. Interestingly, at baseline, 2 of 20 patients were completely asymptomatic (clinical symptoms score $=0$ ), despite the evidence of histologically active disease. The same was observed for another 3 patients at the end of study. All these patients were symptomatic at the time of the diagnosis.

The endoscopy score significantly decreased from 4.25 (range $0-6)$ to 0.4 (range $0-2)(p<0.01)$, and to 0.55 (range $0-3)(p<0.01)$ at 12 and 24 weeks, respectively. At 36 weeks, it increased to 1.8 (range $0-5)(p<0.01)$ (Fig. 4b).

Finally, the histology score changed from 9.2 (range 6-12) to 1.15 (range $0-7$ ) and to 1.6 (range $0-10$ ), at 12 and 24 weeks, respectively ( $p<0.01$ ) (Fig. 4c). At 36 weeks, the mean score was 5.7 (range 0-12). A complete histological remission (histology score $=0$ ) was observed in 11 patients after the induction treatment, but only in 8 at the end of the maintenance period.

No patient reported serious adverse events during the study period. No cases of candidiasis (either oral or esophageal) were described.

There was no significant difference between pre-/postinduction morning cortisol levels, $13.4 \mu \mathrm{g} / \mathrm{dl}$ (range 5.2-23) and $11.1 \mu \mathrm{g} / \mathrm{dl}$ (range 4.5-17), respectively. A similar value, $12.8 \mu \mathrm{g} / \mathrm{dl}$ (range 4.7-19), was described at the end of the maintenance phase. Neither clinically significant drugrelated effects on heart rate or temperature, nor changes in mean height or weight occurred. All patients were considered adherent at the end of treatment, with $97 \%$ (range 96-105) of the expected amount of medication consumed during the 24 weeks of treatment. 
Table 3 Pre- and post-treatment characteristics (before induction and at the end of maintenance) compared with those in remission of patients with a relapse

\begin{tabular}{|c|c|c|c|c|c|c|}
\hline & \multicolumn{2}{|c|}{ Relapse $(n=11)$} & \multicolumn{2}{|c|}{ Remission $(n=9)$} & \multicolumn{2}{|c|}{$P$ value } \\
\hline & Pre & Post & Pre & Post & Pre & Post \\
\hline \multicolumn{7}{|l|}{ Endoscopic findings $[n(\%)]$} \\
\hline Normal & $0(0)$ & $6(55)$ & $0(0)$ & $8(89)$ & NS & 0.09 \\
\hline Rings & $4(36)$ & $1(9)$ & $3(33)$ & $1(11)$ & NS & NS \\
\hline Exudates & $10(91)$ & $1(9)$ & $7(78)$ & $1(11)$ & NS & NS \\
\hline Furrows & $8(73)$ & $3(27)$ & $7(78)$ & $1(11)$ & NS & NS \\
\hline Edema & $4(37)$ & $2(18)$ & $5(56)$ & $1(11)$ & NS & NS \\
\hline Stricture & $0(0)$ & $0(0)$ & $0(0)$ & $0(0)$ & NS & NS \\
\hline Total EREFS (mean \pm SD) & $4.4 \pm 1.2$ & $0.8 \pm 1.2$ & $4.1 \pm 1.3$ & $0.1 \pm 0.3$ & NS & 0.09 \\
\hline Symptoms score $($ mean $\pm \mathrm{SD})$ & $5.1 \pm 2.8$ & $1.1 \pm 1.9$ & $3.4 \pm 2.5$ & $0.1 \pm 0.3$ & NS & NS \\
\hline Peak eosinophil count (mean \pm SD) & $46.8 \pm 13.1$ & $4.7 \pm 7.4$ & $52.7 \pm 25.8$ & $0.3 \pm 1$ & NS & 0.09 \\
\hline Histology score (mean $\pm \mathrm{SD})$ & $9.5 \pm 1.6$ & $2.5 \pm 2.7$ & $8.9 \pm 1.7$ & $0.6 \pm 1.1$ & NS & 0.08 \\
\hline Compliance (mean \%) & - & 95 & - & 97 & - & NS \\
\hline
\end{tabular}

Fig. 4 Median values and IQR of symptom score (a), endoscopy score (b), and histology score (c) during the study period, $* p<0.01$
Symptoms

A

Endoscopy

B
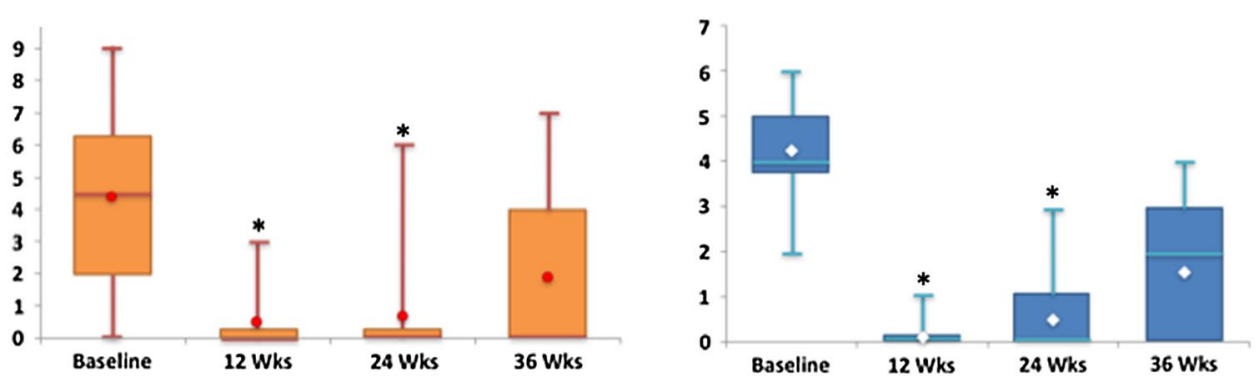

Histology

C

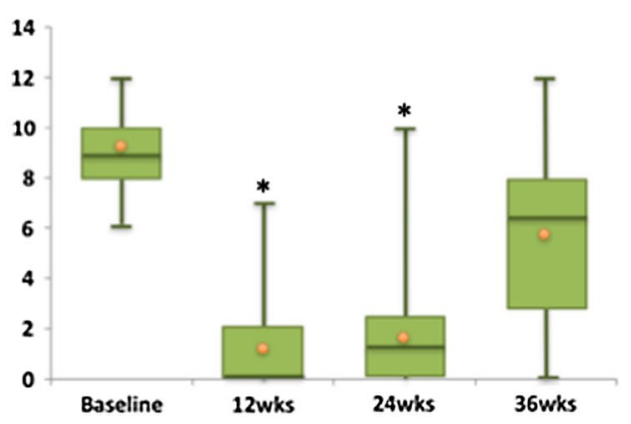

\section{Discussion}

This pilot study confirms the efficacy of the new PVB suspension in inducing and maintaining remission of pediatric EoE for 24 weeks. This formulation was successful in sustaining symptomatic, endoscopic, and histological remission with good tolerability and safety. Noticeably, half of the patients experienced a recurrence at 12 weeks after the treatment cessation.

Remission occurred in $\geq 90 \%$ of children with EoE receiving the PVB suspension. Only two children were partial responders; however, a dose reduction after 12 weeks was considered appropriate for them, due to the symptom and histological improvements. Unfortunately, the dose reduction without reaching complete remission ( $<6$ eos/HPF) led to an increased peak eosinophil count at the subsequent endoscopy, confirming that a deeper reduction in eosinophil esophageal count before halving therapies may be desirable to sustain remission over time.

This study also confirms the ability of PVB to improve several histological findings (i.e., eosinophilic degranulation, eosinophil microabscesses, basal layer hyperplasia dilated intracellular spaces or spongiosis, and lamina 
propria fibrosis). A significant reduction in the histology score was observed during and after treatment, confirming the view that a maintenance treatment in children is crucial [12]. Future studies on longer therapies would be necessary to confirm this outcome.

Improvement in endoscopy and symptoms scores also supports the efficacy of dose reduction. Nevertheless, the high number of flare-ups observed at the end of therapy confirms the chronic nature of the disease. As in our first study [18], half of the patients experienced a histological relapse 12 weeks after the end of treatment, highlighting the concept that EoE is a chronic condition and a maintenance therapy with the lowest pharmacologic dose would be necessary may be desirable to support remission over time [10].

We did not identify any clinical or histological data able to predict sustained remission after interruption of treatment with steroids. No differences in baseline characteristics occurred between children in remission at the end of the study and those with active disease. This outlines the view that different relapse tendencies without baseline dissimilarities reflect different phenotypes in terms of responsiveness to drugs and disease behavior [22].

Two small differences were observed at the end of treatment, in regard to the EREFS value and peak eosinophil count. Lower values seem associated with a higher possibility to maintain remission over time. Unfortunately, this difference was not significant, probably due to the small sample size $(p=0.09)$.

However, it is not yet settled if response to drugs or diet is durable or whether routine surveillance endoscopy is necessary. Interestingly, 3 of our patients had a histological relapse and no specific symptoms at 36-week follow-up. Currently, endoscopy with biopsies still represents the most reliable tool for an appropriate follow-up [23]. Likewise, as recently described, in this study the EREFS score confirmed its good correlation with histology even in childhood [24].

Longer treatment with topical steroids appears to be safe [10], and no severe adverse events were observed in our children over the study period. Indeed, no significant changes in cortisol levels were reported. However, although the morning cortisol assessment should always be considered, it might be not enough in some cases. The use of ACTH stress test may increase the identification of the hidden suppressions, but to date, no symptomatic cases have been reported. Future studies should identify the risk factors for adrenal axis suppression associated with clinical manifestations and not only to biochemical signals [25-28].

Obviously, this study has several limitations. First is the lack of a group of control. However, considering the different concerns currently rising in the scientific community about the use of placebo in pediatric chronic diseases, we preferred to avoid such comparison [29]. Second, we used a higher dosage compared to the common clinical practice.
Our excellent results might be related also to this higher dosage rather than the efficacy of the formulation itself. However, we decided to maintain the same dose protocol we used in the first pilot study on induction [18] to better compare the results between different time points. Obviously, the use of lower dosage of this formulation should be equally considered and studied. Finally, it is also true that the optimal dose is unknown yet.

Third, this study was performed before considering PPI as one of the therapies of EoE. In our cohort, all patients with positive results at MII-pH have received concomitant PPI therapy to reduce the potential effect of concurrent GERD on esophageal eosinophilia. However, the dose of PPI used was not curative for EoE ( $1 \mathrm{mg} /$ day) but only for GERD. As in other series [19], also in this cohort it seems that the low dose of PPI does not influence the efficacy of topical steroids on esophageal eosinophilia in PPI non-responders. However, it is still unclear the actual value of concomitant therapy with PPI and topical steroids, since it has been demonstrated that PPIs have an anti-inflammatory effect [30]. Fourth, we used non-standardized scores for histology and symptoms (except for the EREFS). Despite this concerns many randomized, placebo-controlled studies in children and adults $[19,21]$, nowadays, there are new validated scales that, if used, would have strengthened the study. Unfortunately, at the time of the study these tools were very recently usable, and we have preferred to set the protocol according to our first pilot study, using the same scores in which investigators were more familiar.

Moreover, though neither histology nor endoscopy scores were blinded, the variability of evaluations was reduced having single operators for endoscopy (SO) and histology (AT) in all patients.

In conclusion, this study confirmed that this new PVB suspension is effective and safe to induce and support remission for 24 weeks. The $50 \%$ dose reduction seems to be an effective strategy to maintain a decreased peak eosinophil count in all esophageal levels with reduction in clinical, endoscopic and histological scores. Unfortunately, around $50 \%$ of patients relapsed 12 weeks after the end of therapy. Larger controlled clinical trials would provide more information about dosing, efficacy, and very long-term safety of this new formulation specifically designed for EoE.

Author's contribution Salvatore Oliva contributed to the study concept and design, performed endoscopy procedures, and wrote the manuscript. He is also the guarantor of the article. Danilo Rossetti contributed to the study design, analysis and interpretation of data, and critical revision of the manuscript. Danila Volpe, Cosimo Ruggiero, Debora Vezzoli, and Giusy Russo enrolled and followed up patients, and acquired clinical data. Saverio Mallardo and Sara Isoldi performed MII-pH tests, enrolled and followed patients, 
and acquired clinical data. Antonio Tiberti performed histological assessments of biopsies and contributed to draft the manuscript. Paola Papoff performed sedation for endoscopy. Salvatore Cucchiara is the Head of the Pediatric Gastroenterology Unit and contributed to the study design and supervision and to critical revision of the manuscript. All authors approved the final version of the manuscript.

\section{Compliance with ethical standards}

Conflict of interest ITC Farma provided the entire budesonide supply used in the study. Apart from the provision of the drug, no financial support from the company was received for this study. All authors have no conflict of interest to declare.

Open Access This article is distributed under the terms of the Creative Commons Attribution-NonCommercial 4.0 International License (http://creativecommons.org/licenses/by-nc/4.0/), which permits any noncommercial use, distribution, and reproduction in any medium, provided you give appropriate credit to the original author(s) and the source, provide a link to the Creative Commons license, and indicate if changes were made.

\section{References}

1. Furuta GT, Katzka DA. Eosinophilic esophagitis. $N$ Engl J Med. 2015;373:1640-1648.

2. Dellon ES, Hirano I. Epidemiology and natural history of eosinophilic esophagitis. Gastroenterology. 2018;154:319-332.

3. Chehade M, Sampson HA, Morotti RA, et al. Esophageal subepithelial fibrosis in children with eosinophilic esophagitis. J Pediatr Gastroenterol Nutr. 2007;45:319-328.

4. Dellon ES, Kim HP, Sperry SL, et al. A phenotypic analysis shows that eosinophilic esophagitis is a progressive fibrostenotic disease. Gastrointest Endosc. 2014;79:577-585.

5. Straumann A, Katzka DA. Diagnosis and treatment of eosinophilic esophagitis. Gastroenterology. 2018;154:346-359.

6. Lieberman JA, Morotti RA, Konstantinou GN, et al. Dietary therapy can reverse esophageal subepithelial fibrosis in patients with eosinophilic esophagitis: a historical cohort. Allergy. 2012;67:1299-1307.

7. Liacouras CA, Spergel JM, Ruchelli E, et al. Eosinophilic esophagitis: a 10-year experience in 381 children. Clin Gastroenterol Hepatol. 2005;3:1198-1206.

8. Arias A, Gonzalez-Cervera J, Tenias JM, et al. Efficacy of dietary interventions for inducing histologic remission in patients with eosinophilic esophagitis; a systematic review and meta-analysis. Gastroenterology. 2014;146:1639-1648.

9. Lucendo AJ. Meta-analysis-based guidance for dietary management in eosinophilic esophagitis. Curr Gastroenterol Rep. 2015; 17:464

10. Philpott H, Dellon ES. The role of maintenance therapy in eosinophilic esophagitis: who, why, end how? J Gastroenterol. 2018;53:165-171.

11. Butz BK, Wen T, Gleich GJ, et al. Efficacy, dose reduction, and resistance to high-dose fluticasone in patients with eosinophilic esophagitis. Gastroenterology. 2014;147:324-333.

12. Andreae D, Hanna MG, Magid MS, et al. Swallowed fluticasone propionate is an effective long-term maintenance therapy for children with eosinophilic esophagitis. Am J Gastroenterol. 2016;111:1187-1197.

13. Straumann A, Conus S, Degen L, et al. Long-term budesonide maintenance treatment is partially effective for patients with eosinophilic esophagitis. Clin Gastroenterol Hepatol. 2011;9:400-409.

14. Greuter T, Bussmann C, Safroneeva E, et al. Long-term treatment of eosinophilic esophagitis with swallowed topical corticosteroids: development and evaluation of a therapeutic concept. Am J Gastroenterol. 2017;112:1527-1535.

15. Dellon ES, Sheikh A, Speck O, et al. Viscous topical is more effective than nebulized steroid therapy for patients with eosinophilic esophagitis. Gastroenterology. 2012;143:321-324.

16. Murali AR, Gupta A, Attar BM, et al. Topical steroids in eosinophilic esophagitis: systematic review and meta-analysis of placebo controlled randomized clinical trials. J Gastroenterol Hepatol. 2016;31:1111-1119.

17. Rubinstein E, Lee JJ, Fried A, et al. Comparison of 2 delivery vehicles for viscous budesonide to treat eosinophilic esophagitis in children. J Pediatr Gastroenterol Nutr. 2014;59:317-320.

18. Oliva S, Rossetti D, Papoff P, et al. a new formulation of oral viscous budesonide in treating paediatric eosinophilic oesophagitis: a pilot study. J Pediatr Gastroenterol Nutr. 2017;64:218-224.

19. Gupta SK, Vitanza JM, Collins MH. Efficacy and safety of oral budesonide suspension in paediatric patients with eosinophilic esophagitis. Clin Gastroenterol Hepatol. 2015;13:66-76.

20. Hirano I, Moy N, Heckman MG, et al. Endoscopic assessment of the oesophageal features of eosinophilic oesophagitis: validation of a novel classification and grading system. Gut. 2013;62:489-495.

21. Dohil R, Newbury R, Fox L, et al. Oral viscous budesonide is effective in children with eosinophilic esophagitis in a randomized, placebo-controlled trial. Gastroenterology. 2010;139:418-429.

22. Atkins D, Furuta G, Liacouras T, et al. Eosinophilic esophagitis phenotypes: ready for the prime time? Pediatr Allergy Immunol. 2017;28:312-319.

23. Safroneeva E, Straumann A, Coslovsky M, et al. Symptoms have modest accuracy in detecting endoscopic and histologic remission in adults with eosinophilic esophagitis. Gastroenterology. 2016;150:581-590.

24. Wechsler JB, Bolton S, Amsden K, et al. Eosinophilic esophagitis reference score accurately identifies disease activity and treatment effects in children. Clin Gastroenterol Hepatol. 2017. https://doi. org/10.1016/j.cgh.2017.12.019.

25. Golekoh MC, Hornung LN, Mukkada VA, et al. Adrenal Insufficiency after chronic swallowed glucocorticoid therapy for eosinophilic esophagitis. J Pediatr. 2016;170:240-245.

26. Harel S, Hursh BE, Chan ES, et al. Adrenal suppression in children treated with oral viscous budesonide for eosinophilic esophagitis. J Pediatr Gastroenterol Nutr. 2015;61:190-193.

27. Philla KQ, Min SB, Hefner JN, et al. Swallowed glucocorticoid therapy for eosinophilic esophagitis in children does not suppress adrenal function. J Pediatr Endocrinol Metab. 2015;28:1101-1106.

28. Hsu S, Wood C, Pan Z, et al. Adrenal insufficiency in pediatric eosinophilic esophagitis patients treated with swallowed topical steroids. Pediatr Allergy Immunol Pulmonol. 2017;30:135-140.

29. Turner D, Koletzko S, Griffiths AM, et al. Use of placebo in paediatric inflammatory bowel diseases: a position paper from ESPGHAN, ECCO, PIBDnet, and the Canadian children IBD network. J Pediatr Gastroenterol Nutr. 2016;62:183-187.

30. Molina-Infante J, Bredenoord AJ, Cheng E, et al. Proton pump inhibitor-responsive oesophageal eosinophilia: an entity challenging current diagnostic criteria for eosinophilic oesophagitis. Gut. 2016;65:524-531. 